\title{
Deciphering, communicating, and engineering the CRISPR PAM
}

\author{
Ryan T. Leenay and Chase L. Beisel* \\ North Carolina State University \\ Department of Chemical and Biomolecular Engineering \\ Raleigh, NC 27695-7905
}

*Correspondence to cbeisel@ ncsu.edu (C.L.B.)

Keywords: Cas9, Cpf1, CRISPR-Cas systems, PFS, rPAM 


\section{ABSTRACT}

2 Clustered regularly interspaced short palindromic repeat (CRISPR) loci and their flanking

3 CRISPR-associated (cas) genes make up RNA-guided, adaptive immune systems in prokaryotes

4 whose effector proteins have become powerful tools for basic research and biotechnology. While

5 the Cas effector proteins are remarkably diverse, they commonly rely on protospacer-adjacent

6 motifs (PAMs) as the first step in target recognition. PAM sequences are known to vary

7 considerably between systems and have proven to be difficult to predict, spurring the need for

8 new tools to rapidly identify and communicate these sequences. Recent advances have also

9 shown that Cas proteins can be engineered to alter PAM recognition, opening new opportunities

10 to develop CRISPR-based tools with enhanced targeting capabilities. In this review, we discuss

11 the properties of the CRISPR PAM and the emerging tools for determining, visualizing, and

12 engineering PAM recognition. We also propose a standard means of orienting the PAM to

13 simplify how its location and sequence are communicated. 
16 Over the past 11 years, CRISPR (Clustered Regularly Interspaced Palindromic Repeats) loci and

17 their associated Cas (CRISPR-associated) proteins have transitioned from curious prokaryotic

18 immune systems to revolutionary tools for fundamental biomolecular research, biotechnology,

19 agriculture, and medicine [1-5]. A key driver has been the ease by which the effector proteins of

20 these systems can be utilized as programmable nucleases to specifically bind and/or cleave

21 selected DNA or RNA sequences. Because these proteins and their guide RNAs are easier and

22 cheaper to implement than all comparable technologies, CRISPR technologies have become a

23 standard for applications in genome editing, gene regulation, and DNA imaging and are being

24 explored for gene drives and sequence-specific antimicrobials [6-16]. CRISPR-Cas immune

25 systems have also proven to be remarkably diverse, with new and emerging systems poised to

26 further advance existing applications or drive entirely new ones [17-20]. Despite this diversity,

27 CRISPR-Cas systems rely on a common set of rules for target recognition: complementary

28 between the guide RNA and the target sequence, and a protospacer-adjacent motif (PAM)

29 flanking the target [21-29]. This review details the nature of the PAM and recent efforts to

30 identify, disseminate, and alter the recognized PAM sequences for different CRISPR-Cas

31 systems. We describe the field's current understanding of what defines a PAM as well as

32 available methods to identify and communicate these sequences. We also discuss efforts to re-

33 engineer PAM recognition and generate CRISPR-Cas systems with altered or improved DNA

34 recognition capabilities.

35

36 CRISPR Biology

37 CRISPR-Cas systems naturally function as adaptive immune systems that protect bacteria and 
38 archaea from foreign genetic material such as bacteriophages or plasmids. The ability to uniquely

39 recognize foreign sequences stems from the CRISPR array, a short stretch of DNA composed of

40 alternating conserved repeats and target-specific spacers [1,30-32]. Each spacer is directly

41 acquired from a fragment of the foreign genetic material called the protospacer, allowing the

42 CRISPR array to possess heritable memory of the infection [30]. The CRISPR array is

43 transcribed and subsequently processed into individual units called CRISPR RNAs (crRNAs)

44 [33-35]. These RNAs associate with the system's Cas effector proteins to form a

45 ribonucleoprotein surveillance complex. Once assembled, the complex scans the cell for PAM

46 sequences. Upon binding, the complex interrogates the extent of base pairing between the

47 downstream sequence and the spacer portion of the crRNA. Extensive base pairing leads to the

48 Cas proteins cleaving or degrading the target sequence, resulting in the clearance of the foreign 49 invader.

50 CRISPR-Cas systems possess a diverse compilation of genes and are found throughout

51 the prokaryotic world. Current estimates place CRISPR-Cas systems within $\sim 50 \%$ of bacterial

52 genomes and $\sim 90 \%$ of archaeal genomes [18], although recent metagenomics sequencing

53 analyses have suggested that the frequency of CRISPR-Cas systems in nature could be much

54 lower [36]. Furthermore, only a subset of the identified systems may be active. Roughly 100

55 protein families have been associated with these systems, where the varying prevalence and co-

56 occurrence of these genes has spurred the development of numerous classification schemes

$57[18,19,37,38]$. The most recent scheme breaks the systems into two classes, six types, and 19

58 subtypes $[18,19]$. The two classes are differentiated by whether a protein complex (Class 1$)$ or

59 individual protein (Class 2) serves as the effector of immune surveillance. The six types (I - VI)

60 are defined by the presence of a signature gene encoding a protein responsible for nucleic-acid 
61 cleavage (e.g. Cas3 for Type I systems, Cas9 for Type II systems). The types also differ in their

62 mechanisms of crRNA processing and target recognition, as well as whether the target is DNA

63 (Types I, II, V), RNA (Type VI), or both (Type III) [39,40]. The subtypes are named by addition

64 of a letter to the type (e.g. I-A, II-C) and are defined based on the specific cas genes and their

65 configuration. As the vast expanse of CRISPR-Cas systems in the prokaryotic world remain

66 poorly characterized, more unique functions and systems are expected to be discovered and will

67 ultimately yield new CRISPR tools and technologies.

\section{PAM Characteristics}

70 The PAM was first observed in 2008, when Horvath and coworkers noticed conserved sequences

71 that flanked protospacers acquired by Streptococcus thermophilus after being challenged with a

72 lytic bacteriophage [21,22]. The following year, Mojica and coworkers uncovered similar motifs

73 for multiple CRISPR-Cas systems through bioinformatics analyses, which established PAMs as

74 generalized features of these systems [23]. Each report coined different names for these motifs-

75 CRISPR motifs or protospacer-adjacent motifs (PAMs), respectively--where the latter became

76 the accepted terminology.

77 The first insights into the function of these motifs first came from studies of the Type III

78 CRISPR-Cas system in Staphylococcus epidermidis by Marraffini and Sontheimer, who

79 demonstrated that these flanking sequences are essential for self/non-self discrimination (Figure

80 1) [41]. They specifically showed that the system uses these flanking sequences to differentiate

81 between the CRISPR array (self) and the foreign invader (non-self), which both harbor a

82 sequence perfectly complementary to the CRISPR RNA spacer. While the mechanism of

83 discrimination appeared to rely on base pairing between the flanking regions of the spacer and

84 protospacer [41], it established the theme that flanking sequences such as PAMs are critical for 
85 protospacer selection and target recognition. This insight was upheld as others showed that the

86 PAM was an essential element for target recognition and cleavage $[9,42]$.

87 Extensive structural and biochemical analyses have helped reveal how the PAM 88 participates in target recognition [43-47]. Cas effector proteins directly bind the PAM sequence

89 through protein-DNA interactions and subsequently unzip the downstream DNA sequence. The

90 effector proteins then interrogate the extent of base pairing between one strand of the DNA target

91 and the spacer portion of the CRISPR RNA. Sufficient complementarity between the two drives

92 target cleavage. Critically, if a PAM sequence is absent, the effector proteins do not interrogate

93 the downstream sequence even if it is perfectly complementary to the spacer. We refer to this

94 unrecognized sequence as a non-PAM. Aside from its function in immune recognition, the PAM

95 also plays an integral role on spacer acquisition. In this case, the acquisition proteins alone or in

96 coordination with effector proteins recognize defined PAM sequences while acquiring new

97 spacers, ensuring that each new spacer can recognize the invading DNA [48-53]. As PAMs for

98 acquisition and interference can be different [29,54,55], the associated sequences have been

99 respectively termed spacer acquisition motifs (SAMs) and target interference motifs (TIMs)

$100[56,57]$. While this distinction is important, we refer to both motifs as PAMs given our primary

101 focus on immune defense and the limited adoption of the terms SAMs and TIMs.

102 Despite the common role of the PAM in target recognition, its characteristics vary 103 between the different types of CRISPR-Cas systems. One major difference is the location of the 104 PAM. Using the non-target strand of the protospacer as a reference, the PAM is located on the 5' 105 of the protospacer for Type I and V systems and on the 3' end of the protospacer for Type II 106 systems. Note that the target strand also has been used to specify the PAM [29,43,58,59], 107 creating some confusion about the exact location and sequence of any reported PAM; see Box 1 
108 and Figure 2 for more information on these orientations and why we recommend the guide-

109 centric orientation used in this review. Figure 3 illustrates the location of the PAM for different

110 CRISPR-Cas system types given this orientation. In the case of Type III and Type VI systems,

111 limited evidence suggests that the PAM is located within the target RNA $[39,40]$. Because of this

112 unique location, the PAM for these systems was renamed the RNA PAM (rPAM) or the

113 protospacer-flanking sequence (PFS), respectively. Given that Type III systems are thought to

114 rely on base pairing between the crRNA 5' handle and the region flanking the target DNA

115 sequence [41], more work is needed to determine whether this mechanism or the rPAM is

116 normally implemented and whether they occur separately or together.

117 Aside from location, the composition of the PAM can vary widely. The composition

118 includes the sequences comprising the PAM, the length of the linker (represented by N's, where

$119 \mathrm{~N}$ any one of the four possible bases) separating the protospacer and the sequence-specific

120 portion of the PAM, and the promiscuity in deviating from a defined consensus sequence. As one

121 example, the widely used Type II-A system from Streptococcus pyogenes recognizes a NGG

122 PAM, and to a lesser extent, an NAG PAM [9,23,60]. Separately, one Type II-A system from

123 Streptococcus thermophilus recognizes an NNAGAA PAM but has the ability to recognize other

124 sequences such as NNGGAAA and may accommodate changes in its linker length of 2

125 nucleotides [21,22,61,62]. Finally, the Type I-E system from Escherichia coli has one of the

126 most promiscuous PAM recognition capabilities, with at least nine recognized PAM sequences

127 (NAAG, NAGG, NATG, NGAG, NTAG, NAAC, NAAA, NAAT, NATA) and a strong

128 nucleotide preference at the $\mathrm{N}$ position for some of these PAMs [29,58,63,64]. Table 1 contains

129 representative consensus sequences for the most active PAMs for each characterized subtype. 
130 Given that PAMs can vary widely even within a given subtype [21-23,65], more work is needed 131 to fully interrogate the diversity of PAMs in nature.

\section{PAM Determination}

134 The PAM is an essential feature of CRISPR-Cas systems, whether for the biological function of

135 the system or for harnessing the system as a biomolecular tool. Determining the full set of 136 functional PAM sequences, however, has proven difficult. This challenge has spurred the 137 development of multiple methods to determine PAMs (Figure 4). Below we describe the 138 available methods along with their particular advantages and disadvantages. While all of these 139 methods reproduce the same highly active PAM sequences, they can often identify differing sup140 optimal PAMs, which can impact target selection and off-target predictions. Thus, the best 141 option for PAM determination will likely depend on the particular CRISPR-Cas system and its 142 end use.

144 Protospacer identification in silico

145 Mojica and coworkers introduced the first means of identifying PAMs as part of their original 146 observation of this motif [23]. Under this method, each spacer sequence from a natural CRISPR 147 array is subjected to a nucleotide BLAST search for homologous sequences [66]. Strong matches 148 that appear to be derived from bacteriophages or plasmids are compiled, and the flanking 149 sequences are aligned to discern a general motif. This method offers a rapid means of identifying 150 potential functional PAM sequences that can be evaluated experimentally. With the recent 151 development of automated online tools such as CRISPRTarget [67], the analysis can be 152 completed in less than a day. One disadvantage is a strong dependence on available sequencing 153 information, which may not contain the associated invader sequences. Another is the challenge 
154 of deciding whether a BLAST hit represents the true source of the spacer, particularly when

155 mismatches are present between the spacer and the putative protospacer sequence. Third, even if

156 the protospacer is the source of the spacer, some of the protospacers may have accumulated

157 mutations in the PAM. Finally, the PAMs associated with acquisition can represent a subset of 158 those that elicit targeting $[48,49,68]$, giving a narrow impression of the PAMs that elicit

159 interference. For these many reasons, the in silico method represents a convenient starting point 160 but often lacks sufficient sequence information and is less suited to obtain a comprehensive 161 picture of the PAM for a given system.

163 Plasmid clearance in vivo

164 The second and most common method screens for functional PAM sequencing using the natural 165 ability of CRISPR-Cas systems to clear foreign genetic material. This method utilizes an effector 166 protein that is either already present in its native host [59] or is imported into a convenient host 167 such as Escherichia coli [60,61,69]. To generate potential PAM sequences, a randomized 168 nucleotide library is inserted next to a target sequence within a plasmid. Plasmids harboring the 169 PAM library are transformed into the host and then subjected to next-generation sequencing. 170 Any plasmids harboring functional PAM sequences would be cleared by the Cas effector 171 proteins, resulting in a substantially lower frequency in the library. This method has been the 172 most widely used because it recapitulates the natural function of CRISPR-Cas systems and has 173 the potential to comprehensively determine all functional PAM sequences. One disadvantage is 174 that the method employs a negative selection, which requires extensive library coverage to 175 identify the missing sequences. Furthermore, because the screen is in vivo, there is a general limit 176 on the library size, and the Cas proteins must be functionally expressed in a non-native host.

177 Finally, the readout of this method is the frequency of PAM escape, whether by mutating the 
178 target or by promoting DNA repair. This is problematic particularly for less-active PAM 179 sequences that may translate poorly to other CRISPR-based applications, such as genome editing 180 or gene regulation $[54,64,70]$.

181 One recent variation on this method generates a library of guide RNAs that tile along the 182 genome of a lytic bacteriophage [39]. The host harboring the CRISPR-Cas system is transformed 183 with the plasmids harboring the guide-RNA library and is then infected with the bacteriophage. 184 Cells survive if the target location on the bacteriophage genome is flanked by a PAM, resulting 185 in the enrichment of guide RNAs targeting functional PAM sequences. This strategy was applied 186 to elucidate the PFS for the RNA-targeting Type VI effector protein C2c2 using the single187 stranded RNA bacteriophage MS2 [39]. By selecting for cells targeting protospacers flanked by a 188 functional PFS, the screen provided a positive selection. The major limitations of this variation 189 are that the guide-RNA library is much smaller than the equivalent PAM library, not all possible 190 PAMs may be sufficiently represented within the bacteriophage genome, and guide-RNA 191 libraries will be much more expensive to generate than PAM libraries. Therefore, this strategy 192 offers some unique advantages over the traditional method if a sufficiently large library can be 193 generated and the associated costs are acceptable.

\section{DNA cleavage in vitro}

196 In contrast to the in vivo plasmid-clearance methods described above, methods have been 197 developed to determine PAMs under in vitro conditions. These methods involve an in vitro 198 cleavage reaction that combines purified Cas effector proteins, the in vitro-transcribed guide 199 RNAs, and a target DNA library of potential PAM sequences. Following the cleavage reaction, 200 the PAM library is subjected to next-generation sequencing. The reaction can be conducted as a 201 positive screen by ligating adapters to the ends of cleaved library members [70,71], or as a 
202 negative screen by sequencing the intact library members [69]. In vitro methods generally offer

203 numerous advantages particularly over in vivo methods. For instance, the screened library can be

204 multiple orders-of-magnitude larger for in vitro screens than for in vivo screens because there are

205 no limitations from transformation efficiencies or cloning in vitro. Furthermore, in vitro reactions

206 grant exquisite control over the assay conditions, such as component concentrations, reaction

207 temperature, and the reaction time. Finally, the ability to sequence the cleavage products

208 represents a positive screen that can reliably identify functional PAM sequences. One downside

209 is that reconstituting the complete system requires complete knowledge of the required

210 components as well as a protein purification. Another is that the ligation step requires a double-

211 stranded break, which excludes Type I systems that cleave and degrade the target. Finally, assay

212 conditions can deviate from the cellular environment, whether it is the buffer conditions, the

213 relative stoichiometry of Cas effector proteins and targets, or the reaction times. This deviation

214 can yield artificial PAM assignments as recently highlighted when Karvelis and coworkers

215 varied the stoichiometry of Cas9 and the target DNA [71]. These in vitro methods thus provide

216 powerful screens to comprehensively determine PAMs for many CRISPR-Cas systems, although

217 the resulting PAMs may not translate well in vivo.

219 DNA binding in vivo

220 The high-throughput experimental methods described above all relied on target cleavage.

221 However, the ability to generate catalytically-dead Cas effector proteins affords the development

222 of PAM determination methods based on DNA binding [8-10]. We recently reported an in vivo

223 DNA-binding method that utilizes catalytically-dead effector proteins to regulate the expression

224 of GFP in E. coli [64]. As part of the screen, binding by the catalytically-dead effector proteins

225 blocks expression of the LacI repressor, which would otherwise block expression of GFP. Based 
226 on this configuration, cells harboring a functional PAM sequence fluorescence, allowing these

227 cells to be isolated by fluorescence-activated cell sorting and subjected to next-generation

228 sequencing. The unique benefits of this method include a positive screen based on the expression

229 of GFP only in the presence of a functional PAM, and the ability to tune the assay stringency by

230 titrating in the LacI inhibitor IPTG. One limitation of the method is the need to identify and

231 inactivate the catalytic domains of the desired CRISPR-Cas systems, particularly if the systems

232 have not undergone initial characterization. Another is the size of the limited library size that can

233 be screened akin to any in vivo screen. Finally, the absence of nuclease activity can yield PAMs

234 that promote efficient binding but not efficient cleavage, even though the PAMs elucidated to-

235 date using this method closely aligned with those determined by cleavage-based methods

$236[61,63,64,69,71-73]$. The in vivo DNA binding method thus may be best suited for better

237 understanding the biophysics of DNA recognition or in applications centered on DNA binding or

238 gene regulation. PAM determination methods that rely on DNA binding in vitro are also under

239 development that could introduce the advantages of in vitro screens [74].

240

\section{$241 \quad$ PAM Reporting}

242 PAM determination methods often generate a large collection of functional PAM sequences that

243 vary in their extent of enrichment (for positive screens) or depletion (for negative screens). The

244 question is how to best report this information without providing the complete list of all 245 sequences and their associated enrichment or depletion scores. A number of reporting schemes

246 have been described, where each is illustrated in Figure 5 using published PAM determination 247 data for the widely used Type II-A system from S. pyogenes and the well-characterized Type I-E

248 system from Escherichia coli [64,72]. As illustrated in this figure, each reporting scheme 249 manages a trade-off between simplicity and information content. 
251 to date starting with the original discovery of the PAM [21,22]. Sequence logos display the 252 conservation of each base within each position: more conserved or highly active bases appear as 253 larger letters, while less conserved or less active bases appear as smaller letters. Consensus 254 sequences report a single sequence that captures the most dominant set of functional PAM 255 sequences. For instance, the consensus sequence for the S. pyogenes Cas9 is NGG, while the 256 sequence logo shows a small but notable A in the middle position reflecting partial activity of an 257 NAG PAM $[23,60]$. Conversely, the consensus sequence for the promiscuous E. coli I-E system 258 has been reported as AWG (where $\mathrm{W}$ is $\mathrm{A}$ or $\mathrm{T}$ ) while the sequence logo shows other, smaller 259 letters at each of the three positions $[23,58]$. Both schemes are easy to interpret and can be 260 expanded to any sequence length. However, they also sacrifice individual functional PAM 261 sequences and their relative activity. This loss of information is manageable if the PAMs are 262 simple or only the most active functional PAM sequences are required for the final use of the 263 interrogated CRISPR-Cas system, such as for designing highly active guide RNAs. However, 264 these reporting schemes can obscure the true number of target sites that can be targeted by the 265 system or the prediction of off-target effects by discarding or masking less active functional 266 PAMs. One workaround is reporting multiple consensus sequences that are classified as more or 267 less active [61], although this scheme still misses the full range of functional PAM sequences 268 and activities. In cases where the PAM is relatively simple (e.g. NGG for the S. pyogenes Cas9) 269 or the user is only interested in the most active PAMs, then sequence logos and consensus 270 sequences are sufficient.

271 A separate scheme that better captures individual functional PAM sequences and 272 activities can be described as a PAM table (Figure 5B,E). The table is similar to a codon table in 
273 which each base of the codon appears along the edges and each of the 64 cells represents a

274 distinct sequence. In the case of the PAM table, each cell conveys the activity or

275 enrichment/depletion score for that specific PAM sequence. Cells with similar activities or

276 scores can be colored, although the groupings are somewhat arbitrary. The upside of the table is

277 that it displays all PAM sequences and activities in a relatively compact format, as illustrated for

278 PAMs recognized by the $S$. pyogenes Cas9 and by multiple CRISPR-Cas systems present in 279 Pyrococcus furiosus [59,60]. The major downside is that the table is difficult to expand beyond 280 three bases, greatly limiting the size of a PAM library that can be reported.

281 We recently developed a reporting scheme called the PAM wheel that also captures 282 individual sequences and activities that can convey larger PAM libraries [64] (Figure 5C,F). 283 The PAM wheel are derived from interactive Krona plots [75]. Each PAM sequence is read by 284 moving along a radius of the circle, where the accompanying arrow specifies the location of each 285 base in relation to the protospacer. The relative activity of each PAM sequence scales with the 286 size of the radial arc. The outer, black rings designate functional PAM sequences. As a Krona 287 plot, any sector of the wheel can be expanded to better view a defined subset of sequences, such 288 as PAMs that begin with a G. The ability to fully interrogate all possible sequences grants a 289 comprehensive picture of the PAM landscape that is otherwise obscured by the other available 290 reporting schemes. For instance, the PAM wheel revealed a potential two-base linker for the $S$. 291 pyogenes Cas9 or strong base preferences at the -4 position for some functional PAM sequences 292 for the E. coli Type I-E system (Figure 5). The most notable disadvantage is that the PAM wheel 293 can be difficult to interpret, particularly in comparison to the other described methods. There is 294 still room for additional reporting schemes that effectively capture and convey the higher-order 
295 information content of the PAM, and it will be up to the CRISPR research community to settle

296 on a common scheme or set of schemes to convey PAM sequences and activities.

\section{PAM Engineering}

299 While the PAM remains an essential feature for self/non-self discrimination, it also restricts 300 which sequences can be targeted by a given CRISPR-Cas system and impacts the likelihood of 301 off-target effects. Accordingly, there has been intense interest in modifying Cas proteins302 particularly the widely used Cas9 effector proteins - to change the recognized PAM $303[68,72,76,77]$. This interest has been fueled by the growing number of structural studies that have 304 pinpointed the PAM-interacting domains and how these domains interact with the PAM as part 305 of target recognition [78-80]. The PAM-interacting domains are relatively modular, allowing

306 Nishimasu and coworkers to swap these domains between the closely related S. pyogenes Cas9 307 and S. thermophilus CRISPR3 Cas9 proteins, thereby changing each Cas9's PAM recognition 308 (Figure 6) [45]. Rationally mutating the protein residues involved in PAM binding has proven 309 more difficult as illustrated for the S. pyogenes Cas9 [9], although Anders and coworkers 310 successfully changed PAM recognition using a variant of this protein [77].

311 The most successful PAM engineering efforts to-date combined random mutagenesis of 312 the DNA binding residues or the entire protein with a high-throughput dual selection. Kleinstiver 313 and coworkers applied this combination to change PAM recognition for the $S$. pyogenes Cas9 314 and the compact Staphylococcus aureus Cas9. These efforts generated a variant of the $S$. 315 pyogenes Cas9 with only three point mutations (Cas9 VQR) that changed the recognized PAM 316 from NGG to NGA. The new Cas9 exhibited a lower propensity for off-target effects for the 317 selected targets [72] despite more promiscuous PAM recognition [64]. These efforts also 318 generated a variant of the S. aureus Cas9 that relaxed its recognized PAM consensus sequence 
319 from NNGRRT (where R is A or G) to NNNRRT [76]. Relaxing the requirement for a $\mathrm{G}$ did not 320 impact the propensity for off-target effects despite the expectation that a more flexible PAM 321 would reduce its contribution to targeting specificity [76,81]. Applying similar strategies to other

322 Cas effector proteins such as Cpf1 could generate an expanded set of proteins that are no longer 323 limited to their natural PAM sequences, thereby expanding the types of sequences that can be 324 targeted for genome editing and other applications.

Shipman and coworkers recently extended PAM engineering to the acquisition proteins

326 Cas1 and Cas2 from the E. coli Type I-E system [68]. These proteins function together to 327 integrate new spacers into the CRISPR array [82-84]. While the work by Shipman and 328 coworkers sought to utilize these proteins to incorporate synthetic spacers for long-term memory 329 storage, the two proteins were also engineered to relax their PAM recognition requirements. The 330 engineering was performed using error-prone PCR, followed by integrating spacers from non331 canonical PAMs [68]. The resulting variants of Cas1 and Cas2 still slightly preferred an AAG 332 PAM, although to a lesser extent than the wild-type versions. Taken together, these 333 accomplishments highlight the potential for engineering diverse Cas effector proteins and 334 acquisition proteins for tightened, relaxed, or non-native PAM recognition.

\section{PERSPECTIVES}

337 Our knowledge of what comprises a PAM has been overwhelmingly shaped by a few well338 characterized Cas effector proteins. However, these few proteins stand in contrast to the large 339 diversity of systems currently spanning 19 subtypes and thousands of individual proteins $[18,19]$

340 (Table 1). Furthermore, characterization of different Cas9 proteins from II-A systems revealed

341 large variation in the PAM: for instance, those for Cas9 proteins from S. pyogenes (NGG PAM),

342 S. thermophilus (NNAGAA PAM), and S. aureus (NNGRRT PAM). We predict that 
343 characterizing multiple systems within other subtypes has the potential to reveal similarly diverse

344 PAMs. By performing bioinformatics and structural analyses of these effector proteins, we could

345 learn how PAM recognition domains uniquely recognize different PAM sequences, informing

346 future efforts to engineer PAM recognition. Elucidating PAMs within different subtypes could

347 also reveal the full range extent of PAM sequences and lengths present in nature, highlighting

348 which sequences may be more readily accessible through PAM engineering.

349 High-throughput methods have been invaluable tools to determine PAMs for varying

350 CRISPR-Cas systems. One consistent insight from these methods is the plasticity of the PAM.

351 Rather than a static sequence that is either correct or incorrect, the PAM comprises a range of

352 sequences with varying activities. This is highlighted by the Type V-A Cpf1 effector protein

353 from Francisella novicida, which has a reported PAM consensus of NTTN but exhibits clear

354 bias within the first $\mathrm{T}$ and both N's $[64,69,73]$. Another unique insight is the co-dependency

355 between the PAM and the protospacer. Despite the general assumption that these entities operate 356 independently, separate studies with the Type I-E system from E. coli have shown that the

357 relative activity of different PAMs depended on the specific target sequence [54,64]. Whether

358 this co-dependency extends to other systems remains to be explored and may need to become a 359 standard part of any attempt at high-throughput PAM determination. Finally, there is evidence 360 that PAM determination methods can predict slightly different PAMs for the same CRISPR-Cas 361 system. For instance, characterization of the Streptococcus thermophilus CRISPR1 Cas9 using 362 an in vivo plasmid-clearance assay, an in vitro cleavage assay, and an in vivo binding assay 363 revealed different suboptimal PAMs [64,71,72]. While these differences may be attributed to the 364 selected PAM reporting schemes, they highlight how the available PAM determination methods 365 can yield disparate results. These differences can be important, as elucidated PAMs may not be 
universally relevant across all applications. As a result, it may be best to perform PAM

367 determination methods that best align with the final application (i.e. in vitro cleavage assays for 368 genome editing; binding assays for transcriptional regulation).

369 The ability to engineer PAM recognition holds great potential for CRISPR technologies.

370 One potential outcome is revising the process of guide-RNA design. The current process

371 involves identifying a PAM within a genetic locus and then selecting the flanking sequence as

372 the target. However, PAM engineering could be used to generate a collection of effector proteins

373 that together recognize all possible PAM sequences. For instance, the collection could include 15

374 derivatives of the S. pyogenes Cas9 that each recognize a variant of the NGG consensus PAM.

375 Separately, the PAM could be lengthened to recognize a more specific sequence that is likely to

376 be found across a genome. These mutations could be combined with those known to better reject

377 mismatches between the guide RNA and the target [85,86], potentially yielding highly specific

378 Cas effector proteins with negligible off-target effects.

379

380 ACKNOWLEDGMENTS

381 We thank Michelle Luo, Matthew Waller, and Rodolphe Barrangou for constructive feedback on 382 the manuscript. The work was supported by funding from the National Science Foundation 383 (CBET-1403135, MCB-1452902 to C.L.B.), the National Institutes of Health (1R35GM11956138401 to C.L.B., 5T32GM008776-15 to R.T.L.), and Agilent Technologies (to C.L.B.). C.L.B. and 385 R.T.L. have submitted provisional patent applications on CRISPR technologies. C.L.B. is a co386 founder of Locus Biosciences and a member of its scientific advisory board. 


\section{REFERENCES}

[1] R. Barrangou, C. Fremaux, H. Deveau, M. Richards, P. Boyaval, S. Moineau, D.A.

Romero, P. Horvath, CRISPR provides acquired resistance against viruses in prokaryotes, Science. 315 (2007) 1709-1712. doi:10.1126/science.1138140.

[2] J.A. Doudna, E. Charpentier, The new frontier of genome engineering with CRISPRCas9, Science. 346 (2014) 1258096. doi:10.1126/science.1258096.

P.D. Hsu, E.S. Lander, F. Zhang, Development and applications of CRISPR-Cas9 for

[4] J. van der Oost, E.R. Westra, R.N. Jackson, B. Wiedenheft, Unravelling the structural and mechanistic basis of CRISPR-Cas systems., Nat Rev Microbiol. 12 (2014) 479-492. doi:10.1038/nrmicro3279. beyond, Nat. Biotechnol. 34 (2016) 933-941. doi:10.1038/nbt.3659.

P. Mali, L. Yang, K.M. Esvelt, J. Aach, M. Guell, J.E. DiCarlo, J.E. Norville, G.M. Church, RNA-Guided human genome engineering via Cas9, Science. 339 (2013) 823826. doi:10.1126/science.1232033.RNA-Guided. F1000Prime Rep. 5 (2013). doi:10.12703/p5-47.

411 [9] M. Jinek, K. Chylinski, I. Fonfara, M. Hauer, J.A. Doudna, E. Charpentier, A programmable dual-RNA-guided DNA endonuclease in adaptive bacterial immunity, Science. 337 (2012) 816-822. doi:10.1126/science.1225829. 
414 [10] M.L. Luo, A.S. Mullis, R.T. Leenay, C.L. Beisel, Repurposing endogenous type I CRISPR-Cas systems for programmable gene repression., Nucleic Acids Res. 43 (2014) 674-681. doi:10.1093/nar/gku971.

417 [11] K.M. Esvelt, A.L. Smidler, F. Catteruccia, G.M. Church, Concerning RNA-guided gene drives for the alteration of wild populations, Elife. e03401 (2014). doi:10.7554/eLife.03401.

[12] A. Hammond, R. Galizi, K. Kyrou, A. Simoni, C. Siniscalchi, D. Katsanos, M. Gribble, D. Baker, E. Marois, S. Russell, A. Burt, N. Windbichler, A. Crisanti, T. Nolan, A CRISPRCas9 gene drive system targeting female reproduction in the malaria mosquito vector Anopheles gambiae, Nat. Biotechnol. 34 (2016) 78-83. doi:10.1038/nbt.3439.

[13] D. Bikard, C.W. Euler, W. Jiang, P.M. Nussenzweig, G.W. Goldberg, X. Duportet, V.A. Fischetti, L.A. Marraffini, Exploiting CRISPR-Cas nucleases to produce sequencespecific antimicrobials., Nat. Biotechnol. 32 (2014) 1146-1150. doi:10.1038/nbt.3043.

[14] A.A. Gomaa, H.E. Klumpe, M.L. Luo, K. Selle, R. Barrangou, C. Beisel, Programmable removal of bacterial strains by use of genome-targeting CRISPR-Cas systems, MBio. 5 (2014). doi:10.1128/mBio.00928-13.

[15] T. Anton, S. Bultmann, H. Leonhardt, Y. Markaki, Visualization of specific DNA sequences in living mouse embryonic stem cells with a programmable fluorescent CRISPR/Cas system., Nucleus. 5 (2014) 163-172. doi:10.4161/nucl.28488. by an optimized CRISPR/Cas system, Cell. 155 (2013) 1479-1491. doi:10.1016/j.cell.2013.12.001.Dynamic. 
[17] P. Mohanraju, K.S. Makarova, B. Zetsche, F. Zhang, E.V. Koonin, J. Van der Oost,

438

439

440

441

442

443

444

445

446

447

448

449

450

451

452

453

454

455

456

457

458

459
Diverse evolutionary roots and mechanistic variations of the CRISPR-Cas systems, Science. 353 (2016) aad5147. doi:10.1126/science.aad5147.

[18] K.S. Makarova, Y.I. Wolf, O.S. Alkhnbashi, F. Costa, S.A. Shah, S.J. Saunders, R. Barrangou, S.J.J. Brouns, E. Charpentier, D.H. Haft, P. Horvath, S. Moineau, F.J.M. Mojica, R.M. Terns, M.P. Terns, M.F. White, A.F. Yakunin, R.A. Garrett, J. van der Oost, R. Backofen, E. V. Koonin, An updated evolutionary classification of CRISPR-Cas systems, Nat. Rev. Microbiol. 13 (2015) 722-736. doi:10.1038/nrmicro3569.

[19] S. Shmakov, O.O. Abudayyeh, K.S. Makarova, Y.I. Wolf, J.S. Gootenberg, E. Semenova, L. Minakhin, J. Joung, S. Konermann, K. Severinov, F. Zhang, E. V. Koonin, Discovery and functional characterization of diverse class 2 CRISPR-Cas systems, Mol. Cell. 60 (2015) 1-13. doi:10.1016/j.molcel.2015.10.008.

[20] M.L. Luo, R.T. Leenay, C.L. Beisel, Current and future prospects for CRISPR-based tools in bacteria, Biotechnol. Bioeng. 113 (2016) 930-943. doi:10.1002/bit.25851.

[21] P. Horvath, D.A. Romero, A.-C. Coute-Monvoisin, M. Richards, H. Deveau, S. Moineau, P. Boyaval, C. Fremaux, R. Barrangou, Diversity, activity, and evolution of CRISPR loci in Streptococcus thermophilus, J. Bacteriol. 190 (2008) 1401-1412. doi:10.1128/JB.01415-07.

[22] H. Deveau, R. Barrangou, J.E. Garneau, J. Labonte, C. Fremaux, P. Boyaval, D.A. Romero, P. Horvath, S. Moineau, Phage response to CRISPR-encoded resistance in Streptococcus thermophilus , J. Bacteriol. 190 (2008) 1390-1400. doi:10.1128/JB.0141207.

[23] F.J.M. Mojica, C. Diez-Villasenor, J. Garcia-Martinez, C. Almendros, Short motif 
sequences determine the targets of the prokaryotic CRISPR defence system,

461

462

463

464

465

466

467

468

469

470

471

472

473

474

475

476

477

478

479

480

481

482

Microbiology. 155 (2009) 733-740. doi:10.1099/mic.0.023960-0.

[24] J.E. Garneau, M.-È. Dupuis, M. Villion, D. a Romero, R. Barrangou, P. Boyaval, C. Fremaux, P. Horvath, A.H. Magadán, S. Moineau, The CRISPR/Cas bacterial immune system cleaves bacteriophage and plasmid DNA., Nature. 468 (2010) 67-71. doi:10.1038/nature09523.

[25] G. Gasiunas, R. Barrangou, P. Horvath, V. Siksnys, Cas9-crRNA ribonucleoprotein complex mediates specific DNA cleavage for adaptive immunity in bacteria., Proc. Natl. Acad. Sci. U. S. A. 109 (2012) E2579-86. doi:10.1073/pnas.1208507109.

[26] C.R. Hale, P. Zhao, S. Olson, M.O. Duff, B.R. Graveley, L. Wells, R.M. Terns, M.P. Terns, RNA-guided RNA cleavage by a CRISPR RNA-Cas protein complex, Cell. 139 (2009) 945-956. doi:10.1016/j.cell.2009.07.040.

[27] M. Jinek, A. East, A. Cheng, S. Lin, E. Ma, J. Doudna, RNA-programmed genome editing in human cells, Elife. 2013 (2013) 1-9. doi:10.7554/eLife.00471.

[28] M.M. Jore, M. Lundgren, E. van Duijn, J.B. Bultema, E.R. Westra, S.P. Waghmare, B. Wiedenheft, Ü. Pul, R. Wurm, R. Wagner, M.R. Beijer, A. Barendregt, K. Zhou, A.P.L. Snijders, M.J. Dickman, J.A. Doudna, E.J. Boekema, A.J.R. Heck, J. van der Oost, S.J.J. Brouns, Structural basis for CRISPR RNA-guided DNA recognition by Cascade, Nat. Struct. Mol. Biol. 18 (2011) 529-536. doi:10.1038/nsmb.2019.

[29] E.R. Westra, P.B.G. van Erp, T. Künne, S.P. Wong, R.H.J. Staals, C.L.C. Seegers, S. Bollen, M.M. Jore, E. Semenova, K. Severinov, W.M. de Vos, R.T. Dame, R. de Vries, S.J.J. Brouns, J. van der Oost, CRISPR immunity relies on the consecutive binding and degradation of negatively supercoiled invader DNA by Cascade and Cas3., Mol. Cell. 46 
(2012) 595-605. doi:10.1016/j.molcel.2012.03.018.

484

485

486

487

488

489

490

491

492

493

494

495

496

497

498

499

500

501

502

503

504

505

[30] F.J.M. Mojica, C. Díez-Villaseñor, J. García-Martínez, E. Soria, Intervening sequences of regularly spaced prokaryotic repeats derive from foreign genetic elements, J. Mol. Evol. 60 (2005) 174-182. doi:10.1007/s00239-004-0046-3.

[31] R. Jansen, J.D.A. Van Embden, W. Gaastra, L.M. Schouls, Identification of genes that are associated with DNA repeats in prokaryotes, Mol. Microbiol. 43 (2002) 1565-1575. doi:10.1046/j.1365-2958.2002.02839.x.

[32] A. Bolotin, B. Quinquis, A. Sorokin, S. Dusko Ehrlich, Clustered regularly interspaced short palindrome repeats (CRISPRs) have spacers of extrachromosomal origin, Microbiology. 151 (2005) 2551-2561. doi:10.1099/mic.0.28048-0.

[33] J. Carte, R. Wang, H. Li, R.M. Terns, M.P. Terns, Cas6 is an endoribonuclease that generates guide RNAs for invader defense in prokaryotes, Genes Dev. 22 (2008) 34893496. doi:10.1101/gad.1742908.

[34] S.J.J. Brouns, M.M. Jore, M. Lundgren, E.R. Westra, R.J.H. Slijkhuis, A.P.L. Snijders, M.J. Dickman, K.S. Makarova, E. V. Koonin, J. van der Oost, Small CRISPR RNAs guide antiviral defense in prokaryotes, Science. 321 (2008) 960-964. doi:10.1126/science.1159689.

[35] E. Deltcheva, K. Chylinski, C.M. Sharma, K. Gonzales, CRISPR RNA maturation by trans-encoded small RNA and host factor RNase III, Nature. 471 (2011) 602-607. doi:10.1038/nature09886.CRISPR.

[36] D. Burstein, C.L. Sun, C.T. Brown, I. Sharon, K. Anantharaman, A.J. Probst, B.C. Thomas, J.F. Banfield, Major bacterial lineages are essentially devoid of CRISPR-Cas viral defence systems., Nat. Commun. 7 (2016) 10613. doi:10.1038/ncomms10613. 
[37] K.S. Makarova, D.H. Haft, R. Barrangou, S.J.J. Brouns, E. Charpentier, P. Horvath, S. Moineau, F.J.M. Mojica, Y.I. Wolf, A.F. Yakunin, J. van der Oost, E. V. Koonin, Evolution and classification of the CRISPR-Cas systems, Nat. Rev. Microbiol. 9 (2011) 467-477. doi:10.1038/nrmicro2577.

[38] K. Chylinski, K.S. Makarova, E. Charpentier, E. V. Koonin, Classification and evolution of type II CRISPR-Cas systems, Nucleic Acids Res. 42 (2014) 6091-6105. doi:10.1093/nar/gku241. Shmakov, K.S. Makarova, E. Semenova, L. Minakhin, K. Severinov, A. Regev, E.S. Lander, E. V Koonin, F. Zhang, C2c2 is a single-component programmable RNA-guided RNA-targeting CRISPR effector, Science. 353 (2016) aaf5573. doi:10.1101/054742.

J.R. Elmore, N.F. Sheppard, N. Ramia, T. Deighan, H. Li, R.M. Terns, M.P. Terns, Bipartite recognition of target RNAs activates DNA cleavage by the Type III-B CRISPRCas system, Genes Dev. 30 (2016) 447-459. doi:10.1101/gad.272153.115.

\section{L.A. Marraffini, E.J. Sontheimer, Self versus non-self discrimination during CRISPR} RNA-directed immunity, Nature. 463 (2010) 568-571. doi:10.1038/nature08703. doi:10.1073/pnas.1111854108.

Westphal, A.J.R. Heck, E.J. Boekema, M.J. Dickman, J. Douda, RNA-guided complex from a bacterial immune system enhances target recognition through seed sequence interactions, Proc. Natl. Acad. Sci. 108 (2011) 15010-15010.

S. Mulepati, A. Héroux, S. Bailey, Crystal structure of a CRISPR RNA-guided surveillance complex bound to a ssDNA target., Science. 345 (2014) 1479-1484. 
doi:10.1126/science.1256996.

530

531

532

533

534

535

536

537

538

539

540

541

542

543

544

545

546

547

548

549

550

551

[44] M. Jinek, F. Jiang, D.W. Taylor, S.H. Sternberg, E. Kaya, E. Ma, C. Anders, M. Hauer, K. Zhou, S. Lin, M. Kaplan, A.T. Iavarone, E. Charpentier, E. Nogales, J.A. Doudna, Structures of Cas9 endonucleases reveal RNA-mediated conformational activation., Science. 343 (2014) 1247997. doi:10.1126/science.1247997.

[45] H. Nishimasu, F.A. Ran, P.D. Hsu, S. Konermann, S.I. Shehata, N. Dohmae, R. Ishitani, F. Zhang, O. Nureki, Crystal structure of Cas9 in complex with guide RNA and target DNA, Cell. 156 (2014) 935-949. doi:10.1016/j.cell.2014.02.001.

[46] C. Anders, O. Niewoehner, A. Duerst, M. Jinek, Structural basis of PAM-dependent target DNA recognition by the Cas9 endonuclease., Nature. 513 (2014) 569-573. doi:10.1038/nature13579.

[47] S.H. Sternberg, S. Redding, M. Jinek, E.C. Greene, J. Doudna, DNA interrogation by the CRISPR RNA-guided endonuclease Cas9, Nature. 507 (2014) 62-67. doi:10.1038/nature13011.

[48] I. Yosef, D. Shitrit, M.G. Goren, D. Burstein, T. Pupko, U. Qimron, DNA motifs determining the efficiency of adaptation into the Escherichia coli CRISPR array., Proc. Natl. Acad. Sci. U. S. A. 110 (2013) 14396-401. doi:10.1073/pnas.1300108110.

[49] E. Savitskaya, E. Semenova, V. Dedkov, A. Metlitskaya, K. Severinov, High-throughput analysis of type I-E CRISPR/Cas spacer acquisition in E. coli, RNA Biol. 10 (2013) 716725. doi:10.4161/rna.24325.

[50] A. Levy, M.G. Goren, I. Yosef, O. Auster, M. Manor, G. Amitai, R. Edgar, U. Qimron, R. Sorek, CRISPR adaptation biases explain preference for acquisition of foreign DNA, Nature. 520 (2015) 505-510. doi:10.1038/nature14302. 
[51] R. Heler, P. Samai, J.W. Modell, C. Weiner, G.W. Goldberg, D. Bikard, L.A. Marraffini, Cas9 specifies functional viral targets during CRISPR-Cas adaptation, Nature. 519 (2015) 1-16. doi:10.1038/nature14245.

[52] Y. Wei, R.M. Terns, M.P. Terns, M.P. Terns, M.P. Terns, Cas9 function and host genome sampling in type II-A CRISPR-cas adaptation, Genes Dev. 29 (2015) 356-361. doi:10.1101/gad.257550.114.

[53] T. Kunne, S.N. Kieper, J.W. Bannenberg, M. Depken, M. Suarez-diez, S.J.J. Brouns, T. Ku, S.N. Kieper, J.W. Bannenberg, A.I.M. Vogel, W.R. Miellet, M. Klein, Cas3-derived target DNA degradation fragments fuel primed CRISPR adaptation, Mol. Cell. 63 (2016) 852-864. doi:10.1016/j.molcel.2016.07.011.

[55] C. Xue, N.R. Whitis, D.G. Sashital, C. Xue, N.R. Whitis, D.G. Sashital, Conformational control of Cascade interference and priming activities in CRISPR immunity, Mol. Cell. 64

[56] F.J.M. Mojica, C. Díez-Villaseñor, Right of admission reserved, no matter the path, Trends Microbiol. 21 (2013) 446-448. doi:10.1016/j.tim.2013.06.003. (2016) 1-9. doi:10.1016/j.molcel.2016.09.033. identities and functional diversity, RNA Biol. 10 (2013) 891-899. doi:10.4161/rna.23764. Sashital, CRISPR interference and priming varies with individual spacer sequences, Nucleic Acids Res. 43 (2015) 10831-10847. doi:10.1093/nar/gkv1259. 
Sci. U. S. A. 108 (2011) 10098-10103. doi:10.1073/pnas.1104144108.

576 [59] J. Elmore, T. Deighan, J. Westpheling, R.M. Terns, M.P. Terns, DNA targeting by the type I-G and type I-A CRISPR-Cas systems of Pyrococcus furiosus, Nucleic Acids Res. 43 (2015) gkv1140. doi:10.1093/nar/gkv1140.

[60] W. Jiang, D. Bikard, D. Cox, F. Zhang, L.A. Marraffini, RNA-guided editing of bacterial genomes using CRISPR-Cas systems, Nat. Biotechnol. 31 (2013) 233-239. doi:10.1038/nbt.2508.

[61] K.M. Esvelt, P. Mali, J.L. Braff, M. Moosburner, S.J. Yaung, G.M. Church, Orthogonal Cas9 proteins for RNA-guided gene regulation and editing., Nat. Methods. 10 (2013) 1116-1121. doi:10.1038/nmeth.2681.

[62] A.E. Briner, P.D. Donohoue, A.A. Gomaa, K. Selle, E.M. Slorach, C.H. Nye, R.E. Haurwitz, C.L. Beisel, A.P. May, R. Barrangou, Guide RNA functional modules direct Cas9 activity and orthogonality, Mol. Cell. 56 (2014) 333-339. doi:10.1016/j.molcel.2014.09.019.

[63] E.R. Westra, E. Semenova, K.A. Datsenko, R.N. Jackson, B. Wiedenheft, K. Severinov, S.J.J. Brouns, Type I-E CRISPR-cas systems discriminate target from non-target DNA through base pairing-independent PAM recognition., PLoS Genet. 9 (2013) e1003742. doi:10.1371/journal.pgen.1003742.

[64] R.T. Leenay, K.R. Maksimchuk, R.A. Slotkowski, R.N. Agrawal, A.A. Gomaa, A.E. Briner, R. Barrangou, C.L. Beisel, Identifying and visualizing functional PAM diversity across CRISPR-Cas systems, Mol. Cell. 62 (2016) 137-147. doi:10.1016/j.molcel.2016.02.031.

597 [65] F.A. Ran, L. Cong, W.X. Yan, D. a. Scott, J.S. Gootenberg, A.J. Kriz, B. Zetsche, O. 
Shalem, X. Wu, K.S. Makarova, E. V. Koonin, P. a. Sharp, F. Zhang, In vivo genome editing using Staphylococcus aureus Cas9, Nature. 520 (2015) 186-190. doi:10.1038/nature14299.

[66] S.F. Altschul, W. Gish, W. Miller, E.W. Myers, D.J. Lipman, Basic local alignment search 602 tool., J. Mol. Biol. 215 (1990) 403-10. doi:10.1016/S0022-2836(05)80360-2.

603 [67] A. Biswas, J.N. Gagnon, S.J.J. Brouns, P.C. Fineran, C.M. Brown, CRISPRTarget: Bioinformatic prediction and analysis of crRNA targets, RNA Biol. 10 (2013) 817-827. doi:10.4161/rna.24046.

[68] S.L. Shipman, S.L. Shipman, J. Nivala, J.D. Macklis, G.M. Church, Molecular recordings by directed CRISPR spacer acquisition, Science. 1175 (2016) 1-16. doi:10.1126/science.aaf1175.

[69] B. Zetsche, J.S. Gootenberg, O.O. Abudayyeh, I.M. Slaymaker, K.S. Makarova, P. Essletzbichler, S.E. Volz, J. Joung, J. van der Oost, A. Regev, E. V. Koonin, F. Zhang,

613 [70] V. Pattanayak, S. Lin, J.P. Guilinger, E. Ma, J.A. Doudna, D.R. Liu, High-throughput profiling of off-target DNA cleavage reveals RNA-programmed Cas9 nuclease specificity, Cpf1 Is a single RNA-guided endonuclease of a class 2 CRISPR-Cas system, Cell. 163 Nat. Biotechnol. 31 (2013) 839-843. doi:10.1038/nbt.2673.

[71] T. Karvelis, G. Gasiunas, J. Young, G. Bigelyte, A. Silanskas, M. Cigan, V. Siksnys, Rapid characterization of CRISPR-Cas9 protospacer adjacent motif sequence elements, Genome Biol. 16 (2015) 253. doi:10.1186/s13059-015-0818-7.

[72] B.P. Kleinstiver, M.S. Prew, S.Q. Tsai, V. V. Topkar, N.T. Nguyen, Z. Zheng, A.P.W. Gonzales, Z. Li, R.T. Peterson, J.-R.J. Yeh, M.J. Aryee, J.K. Joung, Engineered CRISPR- 
Cas9 nucleases with altered PAM specificities, Nature. 523 (2015) 481-485. doi:10.1038/nature14592.

[73] I. Fonfara, H. Richter, M. Bratovič, A. Le Rhun, E. Charpentier, The CRISPR-associated DNA-cleaving enzyme Cpf1 also processes precursor CRISPR RNA, Nature. (2016) 119. doi:10.1038/nature17945.

[74] E.A. Boyle, J.O.L. Andreasson, M. Lauren, S.H. Sternberg, M.J. Wu, K. Chantal, J.A. Doudna, W.J. Greenleaf, High-throughput biochemical profiling reveals Cas9 off- target binding and unbinding heterogeneity, bioRxiv. (2016). doi:10.1101/059782.

[75] B.D. Ondov, N.H. Bergman, A.M. Phillippy, Interactive metagenomic visualization in a Web browser, BMC Bioinformatics. 12 (2011). doi:10.1186/1471-2105-12-385.

[76] B.P. Kleinstiver, M.S. Prew, S.Q. Tsai, N.T. Nguyen, V. V Topkar, Z. Zheng, J.K. Joung, Broadening the targeting range of Staphylococcus aureus CRISPR-Cas9 by modifying PAM recognition., Nat. Biotechnol. 33 (2015) 1-7. doi:10.1038/nbt.3404. doi:10.1016/j.molcel.2016.02.020.

C. Anders, K. Bargsten, M. Jinek, Structural plasticity of PAM recognition by engineered variants of the RNA-guided endonuclease Cas9, Mol. Cell. 61 (2016) 895-902. C. Ma, S. Wang, D. Wu, Y. Ma, S. Fan, J. Wang, N. Gao, Z. Huang, The crystal structure of Cpf1 in complex with CRISPR RNA, Nature. (2016) 1-16. doi:10.1038/nature17944.

643 [80] R.N. Jackson, S.M. Golden, P.B.G. van Erp, J. Carter, E.R. Westra, S.J.J. Brouns, J. van 
der Oost, T.C. Terwilliger, R.J. Read, B. Wiedenheft, Crystal structure of the CRISPR RNA-guided surveillance complex from Escherichia coli, Science. 345 (2014) 1473-1479. doi:10.1126/science.1256328.

[81] S.Q. Tsai, Z. Zheng, N.T. Nguyen, M. Liebers, V. V Topkar, V. Thapar, N. Wyvekens, C. Khayter, A.J. Iafrate, L.P. Le, M.J. Aryee, J.K. Joung, GUIDE-seq enables genome-wide profiling of off-target cleavage by CRISPR-Cas nucleases, Nat Biotechnol. 33 (2015)

[82] I. Yosef, M.G. Goren, U. Qimron, Proteins and DNA elements essential for the CRISPR adaptation process in Escherichia coli, Nucleic Acids Res. 40 (2012) 5569-5576. doi:10.1093/nar/gks216.

[83] J.K. Nuñez, P.J. Kranzusch, J. Noeske, A. V Wright, C.W. Davies, J.A. Doudna, Cas1Cas2 complex formation mediates spacer acquisition during CRISPR-Cas adaptive

[84] K. a. Datsenko, K. Pougach, A. Tikhonov, B.L. Wanner, K. Severinov, E. Semenova, Molecular memory of prior infections activates the CRISPR/Cas adaptive bacterial immunity system, Nat. Commun. 3 (2012) 945. doi:10.1038/ncomms1937. effects, Nature. 529 (2016) 490-495. doi:10.1038/nature16526. engineered Cas9 nucleases with improved specificity, Science. 351 (2015) 84-88. doi:10.1126/science.aad5227.

666 [87] P. Boudry, E. Semenova, M. Monot, K.A. Datsenko, A. Lopatina, O. Sekulovic, M. 
Ospina-Bedoya, L.-C.F.K. Severinov, B. Dupuy, O. Soutourinaa, Function of the CRISPR-Cas System of the human pathogen Clostridium difficile, MBio. 6 (2015) 1-16. doi:10.1128/mBio.01112-15.Editor.

670 [88] K.C. Cady, J. Bondy-Denomy, G.E. Heussler, A.R. Davidson, G.A. O'Toole, The CRISPR/Cas adaptive immune system of Pseudomonas aeruginosa mediates resistance to naturally occurring and engineered phages, J. Bacteriol. 194 (2012) 5728-5738. doi:10.1128/JB.01184-12.

674 [89] G. Crooks, G. Hon, J. Chandonia, S. Brenner, WebLogo: A sequence logo generator, 675 Genome Res. 14 (2004) 1188-1190. doi:10.1101/gr.849004.1.

676

677 
679 Box 1. Standardizing the orientation of the CRISPR PAM. The PAM sequence flanks the 680 protospacer within the target DNA. Because of the double-stranded nature of DNA, only one 681 strand needs to be reported along with its location relative to the protospacer. To date, both 682 strands have been used to report PAM sequences, where the selected strand often trends with the 683 particular type of CRISPR-Cas system. The problems are that consensus PAM sequences are 684 reported without the orientation, and the use of either strand creates confusion about the exact 685 location of the PAMs. Here, we describe both orientations, which we term target-centric and 686 guide-centric, and argue for the guide-centric orientation to be universally adopted. Both 687 orientations are illustrated in Figure 2. Under the target-centric orientation, the PAM is located 688 on the same strand that base pairs with the guide RNA. In many cases, the PAM on this strand is 689 specifically recognized by the Cas effector proteins [43,58], lending a mechanistic argument to 690 this orientation. The target-centric orientation is regularly employed for Type I systems $691[29,43,58,59]$. Under the guide-centric orientation, the PAM is located on the strand that matches 692 the sequence guide RNA. This match lends to guide-RNA design, where the sequence flanking 693 the identified PAM is used to create the guide portion of the RNA. This orientation is used for 694 Type II and V systems [21-23,61,69]. While the two orientations are equivalent, we propose the 695 universal adoption of the guide-centric orientation: the Cas9 effector proteins from Type II 696 systems are the most widely recognized and published, and the PAMs for these proteins are 697 always reported in the guide-centric orientation. Adopting this orientation would therefore have a 698 smaller impact on the existing body of CRISPR literature. 
702 Figure 1. Function of the CRISPR PAM. CRISPR-Cas systems naturally utilize PAMs to

703 discriminate between self and non-self. The spacer portion of the CRISPR RNA is perfectly 704 complementary to both its own CRISPR array (self) and the protospacer within the foreign 705 invader's genetic material (non-self). The systems differentiate between these two through the 706 flanking PAM that is absent within the CRISPR array (gray) and present within the invader's 707 genetic material (red). Because the PAM is essential for target recognition, the CRISPR-Cas 708 systems will target the invader's genetic material but not the CRISPR array.

710 Figure 2. Two orientations for reporting PAM sequences. The PAM sequence is located within 711 double-stranded DNA, where either strand of the PAM can be reported along with its location 712 relative to the protospacer. Under the target-centric orientation, the PAM is reported from the 713 strand that base pairs with the guide RNA. Under the guide-centric orientation, the PAM is 714 reported from the strand that matches the guide RNA and is used for guide-RNA design.

716 Figure 3. PAM orientation and targets for different types of CRISPR-Cas systems. CRISPR-Cas 717 systems are subdivided into two classes and six types. Representative illustrations of the effector 718 proteins for each type are shown. The dsDNA orientation has been flipped from Figure 2 to 719 accommodate the guide-centric PAM orientation. Note that the effector proteins for the V-B and 720 V-C subtypes require a tracrRNA similar to Type II systems. For Type III and VI systems, the 721 crRNA binds target RNAs. Type III systems cleave transcribed dsDNA due to its proximity to 722 the RNA target [40]. Note that the mechanism by which Type III and VI systems recognize their 
723 nucleic-acid targets are still under investigation. PAM orientations are presented for all systems

724 except for Type IV systems, which remain uncharacterized.

725

726 Figure 4. Methods for PAM determination. (A) In silico PAM determination. A BLAST search

727 of metagenomic databases identifies potential protospacers. Matches found on foreign nucleic

728 acid elements from bacteriophages and plasmids are aligned to elucidate a single consensus

729 sequence. (B) PAM determination through plasmid clearance. Plasmids harboring a library of

730 potential PAM sequences are transformed into cells expressing the Cas proteins and the targeting

731 guide RNA, and functional PAM sequences are identified based on their depletion from the

732 library. (C) PAM determination through bacteriophage clearance. A library of guide RNAs are

733 designed so their targets tile along a lytic bacteriophage genome. Guide RNAs targeting a site

734 flanked by a functional PAM protect the bacteriophage attack, as revealed by sequencing the

735 enriched guide RNAs and mapping their locations and flanking sequences within the

736 bacteriophage genome. (D) In vitro PAM determination through DNA cleavage. A large PAM

737 library is incubated with purified Cas proteins and transcribed crRNAs in a reaction buffer.

738 Functional PAMs within this library are cleaved, and a sequencing adapter (shown in orange)

739 can then be ligated for high-throughput sequencing. Alternatively, intact DNA can be sequenced,

740 revealing PAM sequences that were depleted because of DNA cleavage. (E) In vivo PAM

741 determination through DNA binding. Catalytically-dead effector proteins are targeted to a PAM

742 library upstream of the lac operon regulating GFP expression. If a functional PAM sequence is

743 present, binding by the catalytically-dead effector proteins represses expression of LacI, resulting

744 in de-repression of GFP. Fluorescence cells are then isolated by fluorescent-activated cell sorting

745 and sequenced. 
747 Figure 5. Reporting the CRISPR PAM. (A-C) Visualizing the PAM for the S. pyogenes Type II-

748 A system. The reported PAMs are based on data from the plasmid-clearance method conducted

749 by Kleinstiver and coworkers [72]. (D-F) Visualizing the PAM for the E. coli Type I-E system.

750 The reported PAMs are based on data from the DNA-binding method conducted by Leenay and

751 coworkers [64]. (A) The consensus sequence and sequence logo. The consensus sequence

752 represents a compilation of all highly-active functional PAM sequences. The sequence logo

753 displays the sequence conservation of each base at each position in functional PAM sequences

754 [89]. The red box demonstrates the PAM location assuming a guide-centric orientation (see

755 Figure 2). (B) The PAM table. The table displays the enrichment or depletion scores from the

756 conducted screen for each possible sequence. Higher values represent more active PAMs. PAMs

757 with similar activities are colored. (C) The PAM wheel. Individual sequences are read following

758 a radius of the circle. The arrow shows the orientation of each base in relation to the protospacer.

759 The larger the radian occupied by the sequence, the greater its enrichment or depletion score.

760 The outer ring depicts a common functional PAM sequence. PAM wheels are also available as

761 interactive .html files [64].

762

763 Figure 6. Engineering Cas proteins with altered or relaxed PAM recognition. (A) Changing

764 PAM specificity for the Cas9 effector protein. This protein was engineered to recognizes an

765 alternative PAM but not the original PAM, thereby changing which sequences can be targeted by

766 this Cas9. (B) Relaxing PAM specificity for the Cas1 and Cas2 acquisition proteins. These

767 proteins still recognize the original PAM as well as additional PAMs, thereby broadening the

768 sequences that can be acquired with these proteins. 
A

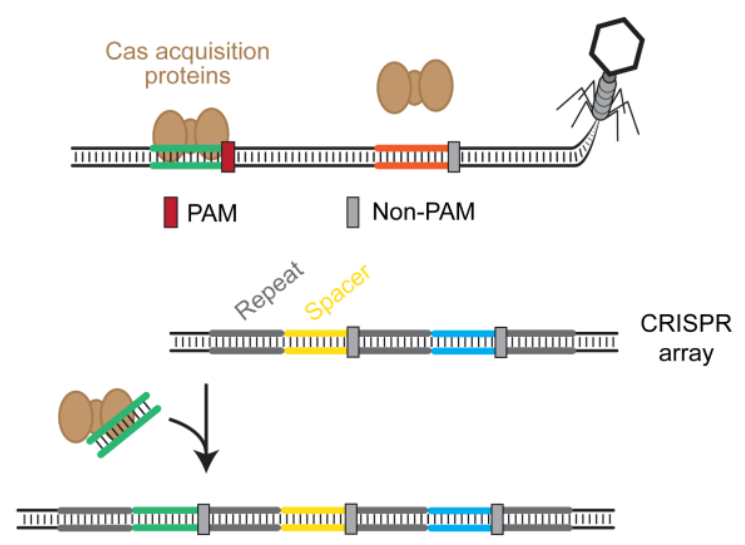

B

Cas effector proteins

\section{1}




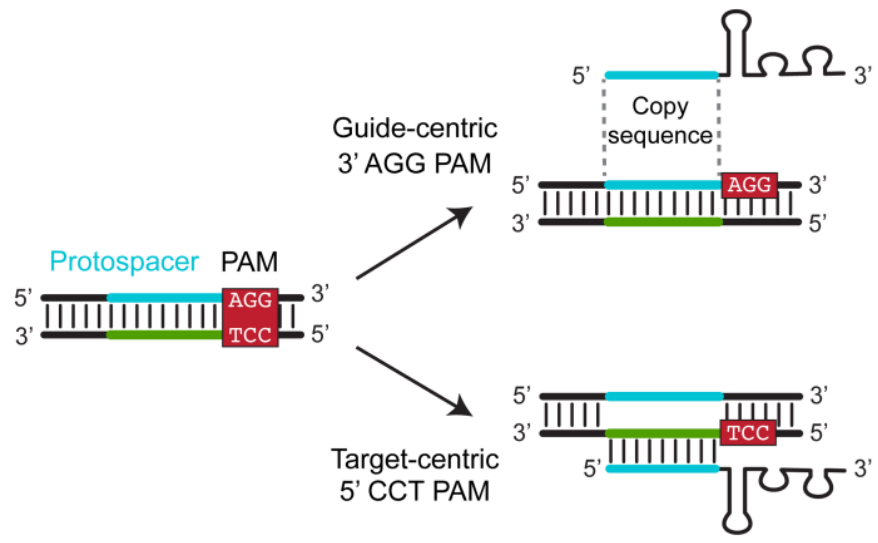



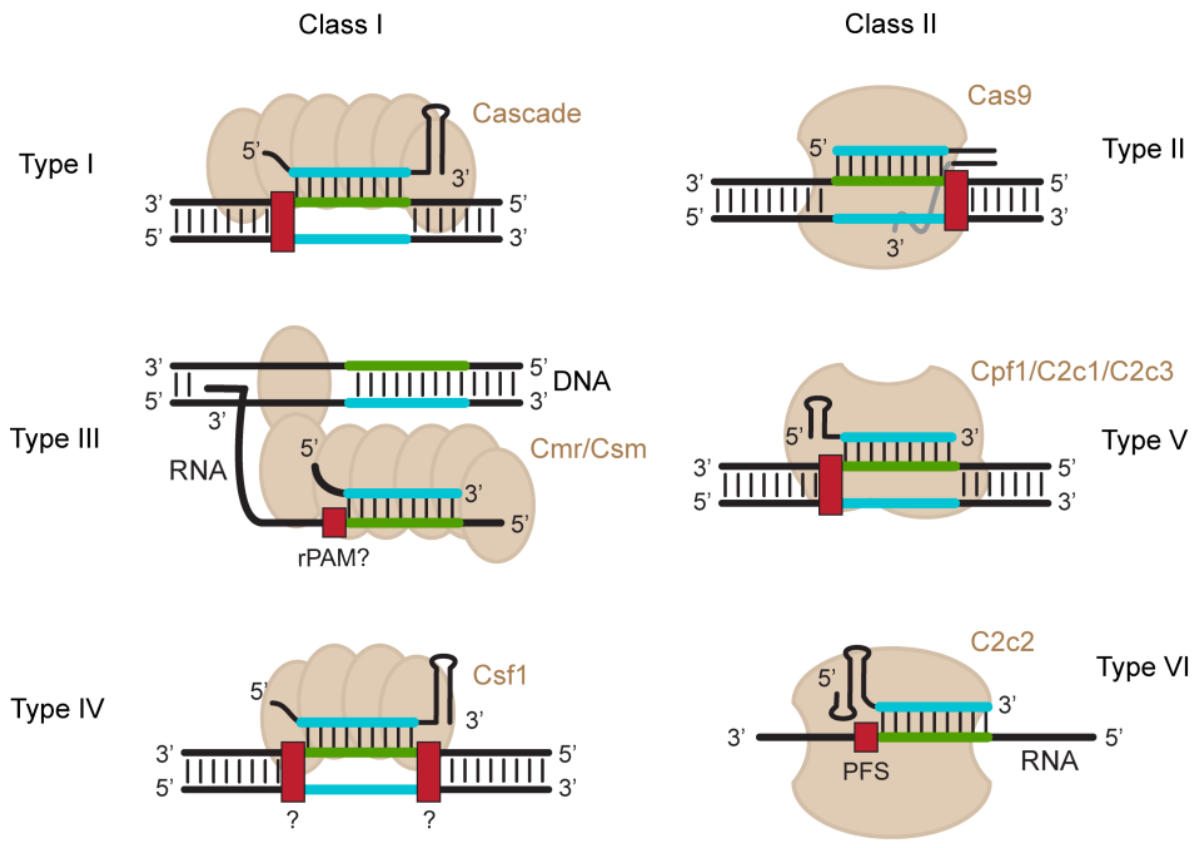
A Identify CRISPR spacers in prokaryotic genome

Find matching foreign protospacers

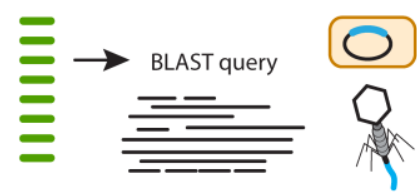

B

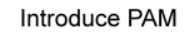

library in plasmids

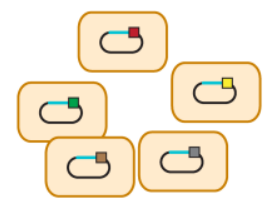

C

Generate tiled guide-RNA library

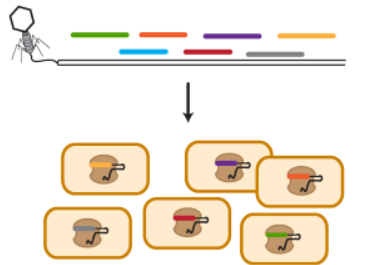

D

Assemble PAM library in vitro

E

Use catalytically dead Cas proteins

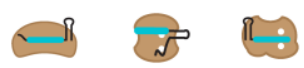

Introduce PAM library in GFP reporter

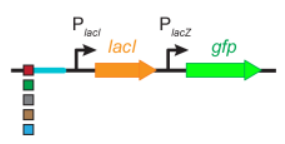

Express Cas machinery, cleave plasmids with PAM
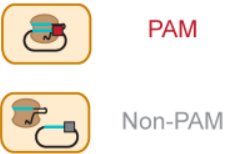

Challenge with lytic phage

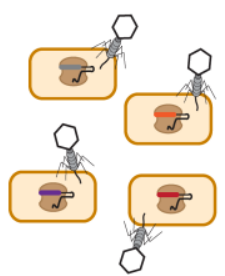

Cleave active PAMs

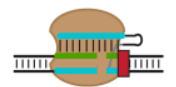

IIIIIIIIIIIIII IIII

Express GFP only for PAMs
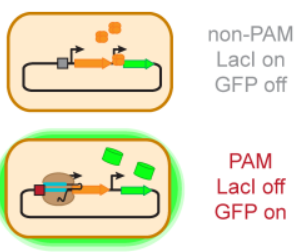

Build consensus PAM

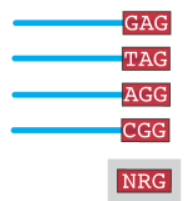

Eliminate cells with PAM, sequence remaining non-PAMs

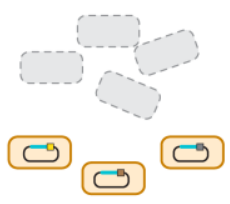

Sequence guide RNAs in survivors, map to phage genome

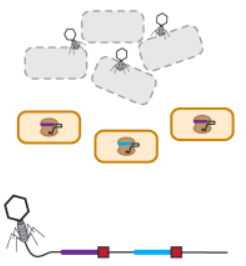

Ligate, sequence cleaved DNA

IIIIIII + IIIIIII $\rightarrow$ IIIIIIIIIIIII Sequencing adapter

Enrich fluorescent cells by FACS

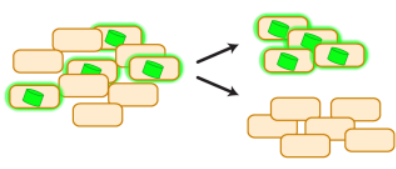


A

S. pyogenes Type II-A Cas9

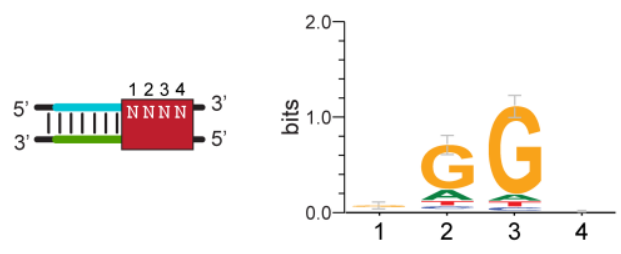

B

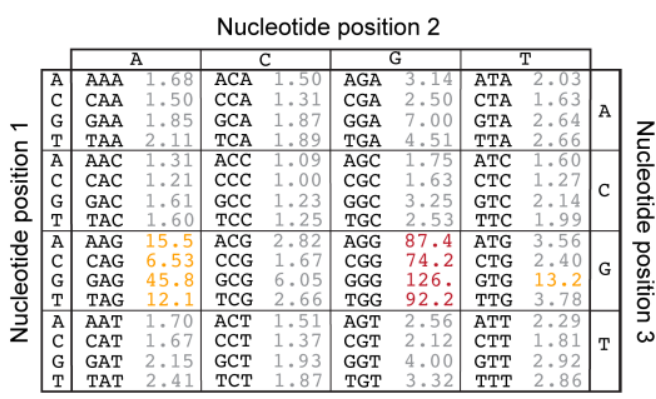

C

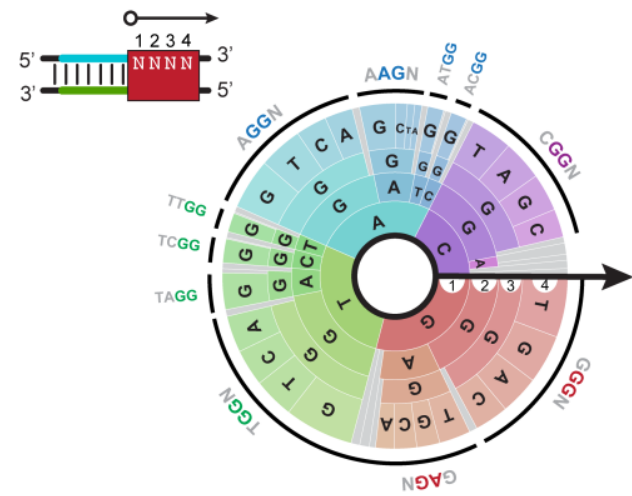

D

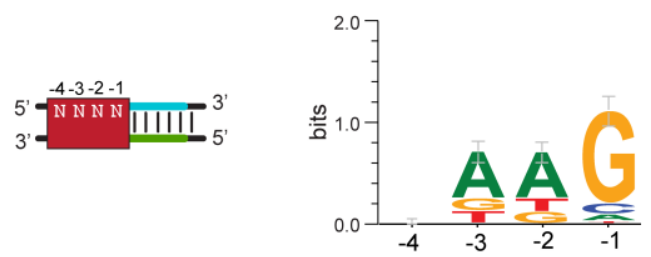

E

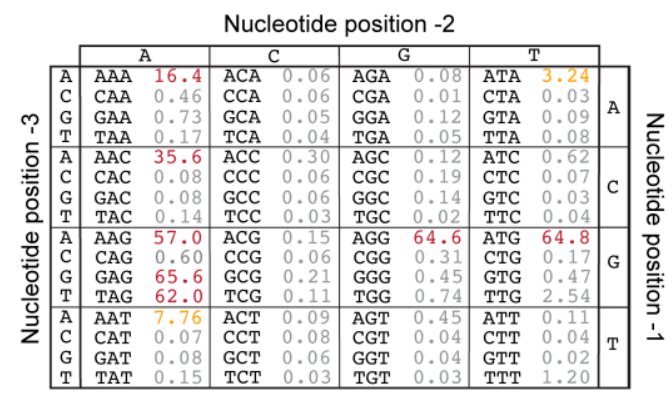

F

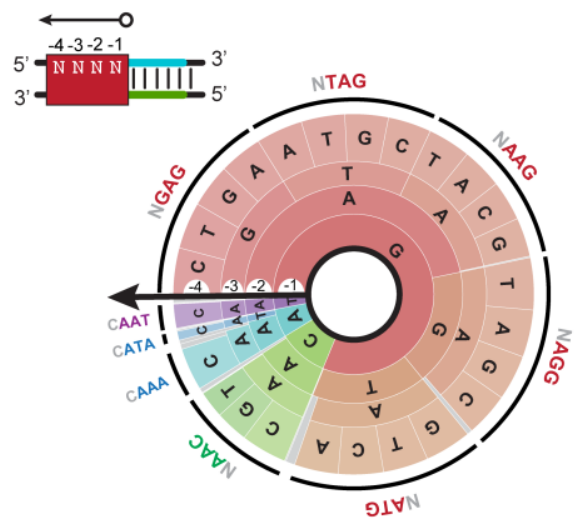


A
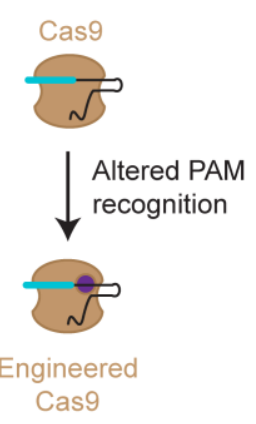

B

780

781
Cas1-Cas2

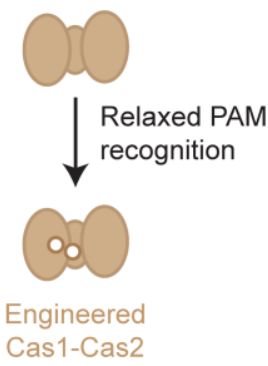

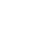

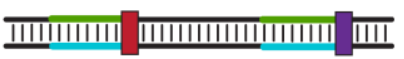

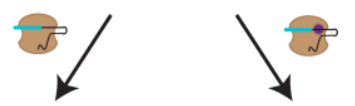

IIII)
III ||I|||||||||||||||||||||||||| ||||||||||||
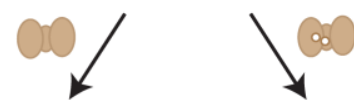

IIIIसम

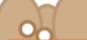

IIIITH

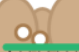

IIIIINH 


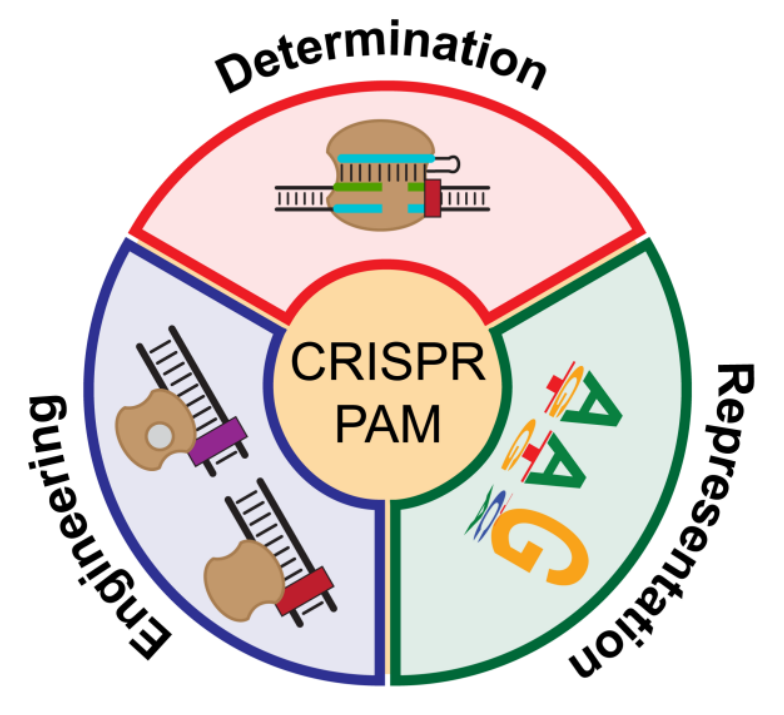

782 